Meta

Journal des traducteurs

Translators' Journal

\title{
La lexicographie assistée par ordinateur. L'expérience d'UZEI
}

\section{Jose Antonio Aduriz et Miriam Urkia}

Volume 42, numéro 2, juin 1997

Lexicologie et terminologie II (1) et Traduction et post-colonialisme en Inde

Translation and Postcolonialism: India (2)

URI : https://id.erudit.org/iderudit/001856ar

DOI : https://doi.org/10.7202/001856ar

Aller au sommaire du numéro

Éditeur(s)

Les Presses de l'Université de Montréal

ISSN

0026-0452 (imprimé)

1492-1421 (numérique)

Découvrir la revue

Citer cet article

Aduriz, J. A. \& Urkia, M. (1997). La lexicographie assistée par ordinateur. L'expérience d'UZEI. Meta, 42(2), 257-263. https://doi.org/10.7202/001856ar

\section{Résumé de l'article}

Cet article présente l'expérience conduite à UZEI concernant l'utilisation des divers outils informatiques dans l'activité lexicographique : l'outil RTerm, créé pour le dépouillement systématique de textes et pour la lemmatisation, l'édition des listes et l'obtention de statistiques ; ORACLE, base de données relationnelle installée sur un système ouvert UNIX ; et EUSLEM, un lemmatiseur automatique, en cours de création, basé sur l'analyse morphologique du basque. 


\title{
LA LEXICOGRAPHIE ASSISTÉE PAR ORDINATEUR. L'EXPÉRIENCE D'UZEI ${ }^{*}$
}

\author{
Jose ANTONIO ADURIZ ET MIRIAM URKIA \\ UZEI, Donostia, Espagne
}

\begin{abstract}
Résumé
Cet article présente l' expérience conduite à UZEI concernant l'utilisation des divers outils informatiques dans l'activité lexicographique : l'outil RTerm, créé pour le dépouillement systématique de textes et pour la lemmatisation, l'édition des listes et l'obtention de statistiques; ORACLE, base de données relationnelle installée sur un système ouvert UNIX; et EUSLEM, un lemmatiseur automatique, en cours de création, basé sur l'analyse morphologique du basque.
\end{abstract}

\section{Abstract}

This article describes UZEI's experience using various computerized tools in lexicographic research. These include Rterm, created for systematic examination of texts, lemmatization, establishing lists and statistics; ORACLE, a relational database in an open UNIX system and EUSLEM, a lemmatiser (under completion) based on a morphological analysis of Basque.

L'UZEI (Centre basque pour la normalisation linguistique) est une association culturelle, créée en 1977, ayant pour but de donner une impulsion à la modernisation du lexique basque dans le processus de normalisation. Trois mots définissent le projet d'UZEI: recherche, normalisation et modernisation de la langue basque, l'euskera.

Au cours des premières années, l'association s'est occupée surtout de terminologie, comme en témoignent les 35 dictionnaires terminologiques multilingues déjà publiés. En 1986, cette activité a atteint sa maturité avec la création d'EUSKALTERM (Centre Basque de Terminologie). EUSKALTERM est aussi une banque de données terminologiques qui peut être consultée par videotex et qui est constamment mise à jour.

En 1987, UZEI a entrepris un nouveau projet: EEBS (Collecte Systématique du Basque Actuel), corpus de 3000000 de mots-textes renouvelé et complété chaque année. Ce projet a été envisagé comme activité complémentaire et plus restreinte au domaine lexicographique. Son but spécifique est de faire connaître l'usage linguistique réel pour ensuite tirer des conclusions sur l'évolution des formes, sur les lacunes à combler, etc.

Pour y parvenir, cinq étapes successives ont été franchies:

1. l'inventaire de ce qui a été publié en basque au $\mathrm{XX}^{\mathrm{e}}$ siècle (livres, revues, journaux) et la classification de chaque unité d'après quatre paramètres : la période ( 3 groupes), la distribution géographique (6 dialectes), la typologie du texte (14 types), la masse textuelle ( 5 groupes);

2. un échantillon de 3000000 de mots-textes a été obtenu selon des critères statistiques pour qu'il soit aussi représentatif que possible de l'univers inventorié (de quelque 300 millions de formes);

3. la mise sur support magnétique des textes statistiquement délimités a été facilitée par le lecteur optique lorsque la qualité du texte le permettait;

4. le dépouillement des mots-textes a été réalisé automatiquement à l'aide de l'outil RTerm;

5. la lemmatisation, par contre, n'a pu être que semi-automatique, pour des raisons que nous expliquerons plus tard.

Meta, XLII, 2, 1997 
Nous avons commencé l'élaboration du corpus sélectionné sur l'outil RTerm, développé par HIZKIA (Bayonne) pour le dépouillement systématique de textes et pour la lemmatisation, l'édition des listes et l'obtention de statistiques. En voici la carte de présentation:

RTerm assiste le lexicographe au cours du travail de dépouillement lexicographique à partir d'un important corpus de textes
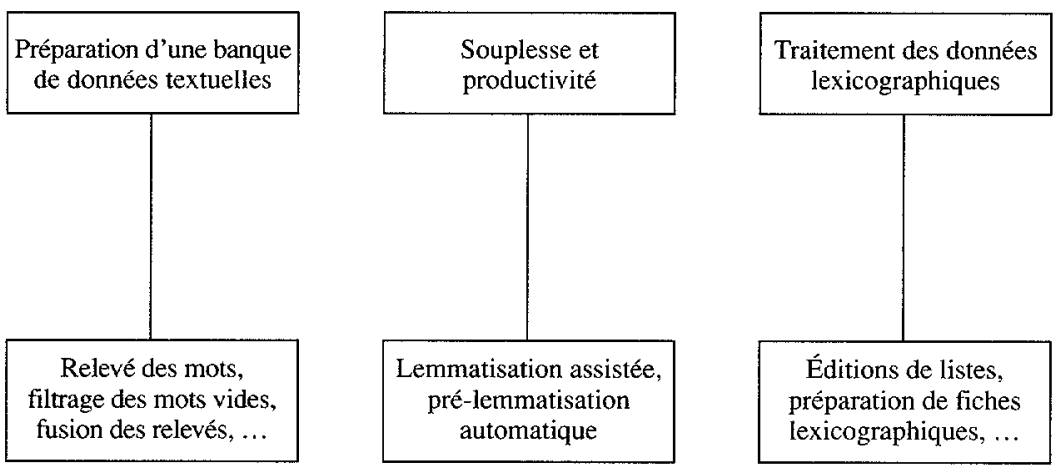

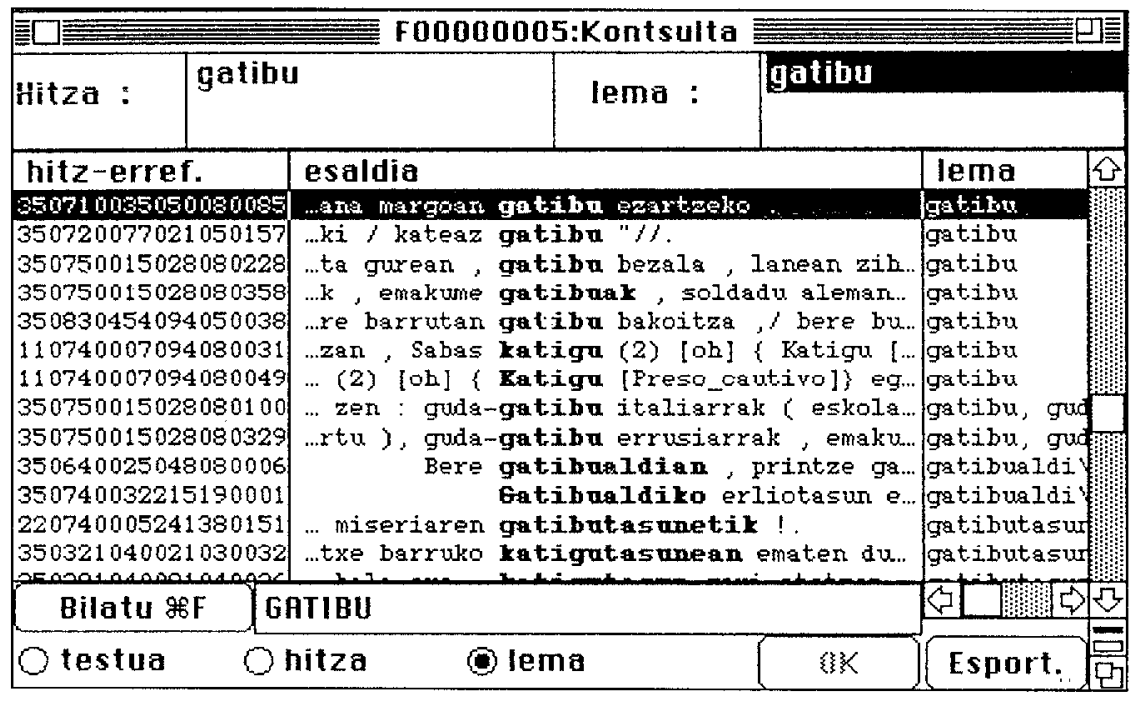


Deux remarques s'imposent sur la typologie et la situation sociolinguistique du basque; elles expliquent la difficulté d'une lemmatisation automatique. Un mot-texte peut se présenter en flexion par le déterminant, le pluralisateur et le cas de déclinaison (par ex. gatibuak («le captif») est composé de la forme lexicale gatibu, le déterminant $a$ et la marque de l'ergatif $k$ ); il peut être aussi l'un des éléments d'un mot composé ou lexie complexe (cf. guda-gatibu), etc. Ajoutons à cela une très large dispersion dialectale et sousdialectale des formes à lemmatiser due, entre autres, à la situation historique particulière du basque, longtemps tenu éloigné de l'enseignement officiel.

Entre 1987 et 1992, UZEI a ainsi créé une base de données de 3 millions de motstextes lemmatisés. Ce corpus va être renouvelé et complété chaque année, afin de pouvoir refléter fidèlement le basque actuel.

Pour sa part, l'Académie de la langue basque (Euskaltzaindia) a créé, en 1992, la Commission du Dictionnaire Unifié et a demandé à UZEI de travailler pour cette commission, chargée tout d'abord de définir un dictionnaire orthographique, comme premier pas vers la normalisation. Afin de fournir à la commission toutes les données significatives pour son travail, UZEI a obtenu une copie du corpus du Dictionnaire Général Basque qui, grâce à ses 5800000 entrées, vise à refléter l'usage classique et traditionnel du basque. Ce deuxième corpus a été mis dans un nouveau système standard, ORACLE.

ORACLE est une base de données relationnelle installée sur un système ouvert UNIX. Nous utilisons trois modules principaux : TextRetrieval (pour l'exploitation des textes: dépouillement, indexation, recherches); SQLforms (communication base de données utilisateur: lemmatisation) et ProC (SQL + langage C: programmes et statistiques).

Étant donné que le corpus du Dictionnaire Général Basque n'est pas préalablement lemmatisé, nous sommes obligés de lemmatiser les formes sur lesquelles la commission nous demande de l'information (la lemmatisation du corpus dans sa totalité reste donc à faire).

En ce qui concerne la lemmatisation à proprement parler, le nouveau système permet d'établir non seulement le lemme standard, mais aussi le lemme de chaque variante, ainsi que sa catégorie grammaticale et les éléments des mots composés.

C'est pour cela que nous avons jugé que le moment était venu de transférer le corpus EEBS de l'outil RTerm à la nouvelle base de données ORACLE qui nous permet le traitement des variantes.

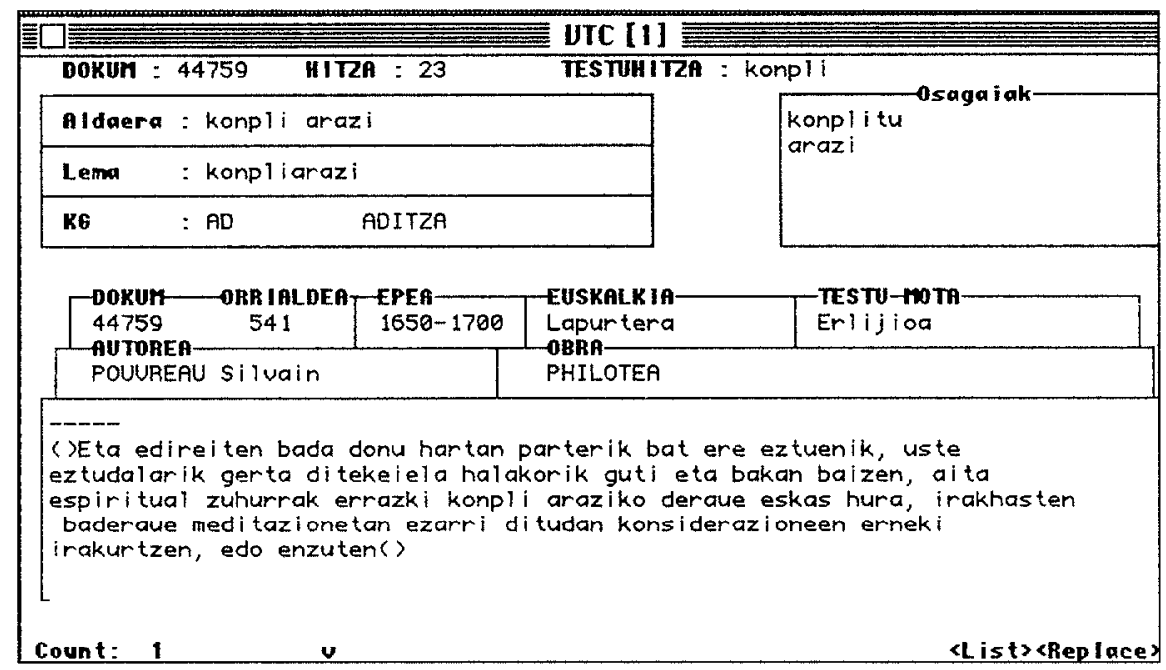


Dès que l'on a lemmatisé les formes à étudier, on a accès directement à l'histoire et à la distribution de chaque forme et de ses variantes. La banque de données est alors prête à fournir des renseignements sur le lemme standard (lema), le lemme de la variable (aldaera), les éléments des mots composés (osagaiak), la catégorie grammaticale $(K G)$ de chaque mot-texte, ainsi que sur sa localisation temporelle et géographique grâce aux indications de l'auteur (autorea), l'œuvre (obra), la période (epea) et le dialecte (euskalkia), sans compter le contexte.

La base de données permet également d'obtenir des cadres synoptiques présentés selon différents critères. Nous n'en retiendrons ici que les deux plus significatifs.

Synopsis de la distribution d'après les auteurs (simplifié) :

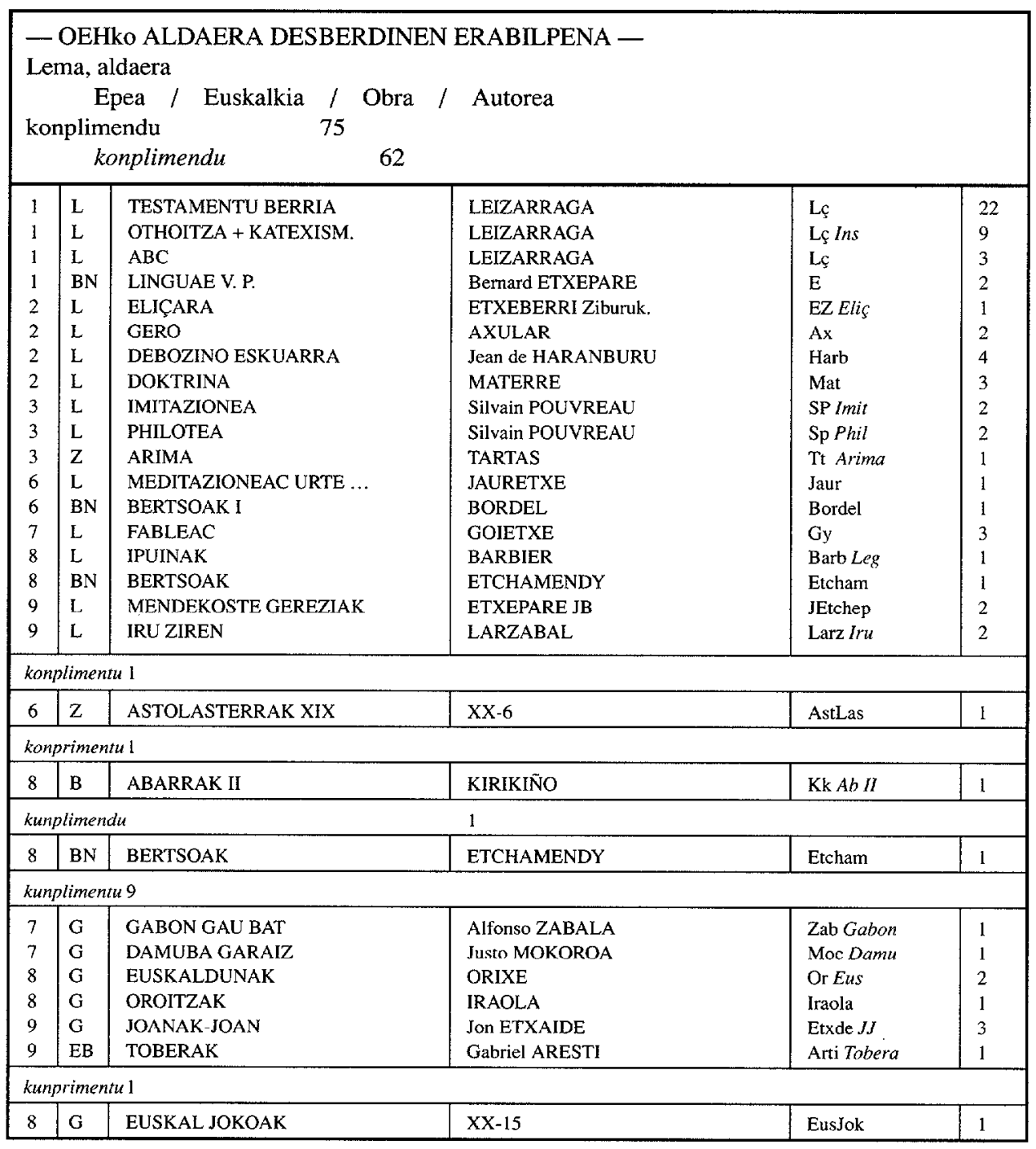


Synopsis des emplois diachronique et dialectal (simplifié):

\begin{tabular}{|c|c|c|c|c|c|c|c|c|c|c|}
\hline & \multicolumn{2}{|c|}{ DGV } & \multicolumn{2}{|c|}{$\begin{array}{c}\text { >>konplitu<< } \\
1-7(<1900)\end{array}$} & & \multicolumn{2}{|c|}{$8-9(>1900)$} & \multirow[b]{2}{*}{$\mathrm{ZuAm}$} & \multirow[b]{2}{*}{ EB } & \multirow[b]{2}{*}{ GUZT } \\
\hline & B & $\mathrm{G}$ & IE & $\mathrm{ZuAm}$ & $\mathrm{B}$ & $\mathrm{G}$ & IE & & & \\
\hline konplimendu & - & 2 & 55 & 2 & 1 & 7 & 7 & - & 1 & 75 \\
\hline konplimendu & - & - & 55 & 1 & - & - & 6 & - & - & 62 \\
\hline konplimentu & 一 & - & - & 1 & - & - & - & - & - & 1 \\
\hline konprimendu & - & - & - & - & 1 & - & - & - & - & 1 \\
\hline konplimendu & - & - & - & - & - & - & 1 & - & - & 1 \\
\hline konplimentu & - & 2 & - & - & - & 6 & - & - & 1 & 9 \\
\hline konprimentu & - & - & - & - & - & 1 & - & - & - & 1 \\
\hline
\end{tabular}

Le portrait d'une forme, avec sa tradition, ses déviations, l'emploi diachronique et l'emploi dialectal, sa distribution d'après les auteurs, etc., devient un instrument de travail fondamental pour la Commission du Dictionnaire Unifié qui, en «lisant» ces données-là, pourra en tirer les conclusions pertinentes pour la normalisation et la standardisation de la langue.

Nous avons développé aussi une base de données lexicographique destinée à formaliser et à garder, dans un format abrégé et codé, toute l'information historique et dialectale sur chaque forme (cf. erabilerak), ainsi que les critères utilisés par la commission dans la lecture qu'elle fait de cette information (cf. azalpena). Cette base de données pourra fournir une base solide pour l'élaboration du dictionnaire normatif de la langue.

Contenu d'un enregistrement:

\begin{tabular}{|l|l|l|l|} 
Format & Kodea & Erabakia & \multicolumn{1}{l}{ Data } \\
\hline erretore & Z1 : BatHizt & B & 94.05 .26 \\
\hline
\end{tabular}

Erabilerak

201 :

2021: 'apaiza' adierakoa bakarrik agertu da: erretor da forma nagusia 408 agerraldirekin (IE: 399; B-G: 8; EB: 1); erretore 141 aldiz azaldu da (B: 5; G; 106, IE: 24; B: 6); ertor (IE: 35) bakarrik.

2022: 'eliza bateko buru den apaiza' adieran erretor 78 aldiz jaso da (B: 1; G: 5 ; IE: 66; EB: 6), erretore 71 (B: 2; G. 21 aldiz (IE); 19, hiru testutan; EB: 20; EgAs: 9) eta ertor 3 aldiz (IE). 'Unibertsitateko burua' adierarekin errektore eta eratorriak (errektoregai, errektoretza) dira nagusi, 13 ager.ekin; eta ager. banarekin: erretore, erretore-orde, erretoreordetza, erretoretza.

Azalpena

3121: erretore da hobestekoa (hots: 'parrokiako arduradun nagusia' adieran), B eta G batetik eta IE bestetik baitabiltza; hori da, gainera, erabilera zainduetan hedatuena (ik. 3133 irizpidea ere).

454: $\quad$ or/-ore.

5332: parrokiako arduradun nagusia; cf. errektore 'Unibertsitateko kargudun nagusia'.

Iritzi-emaileak

Oharra

jatorrizkoan : erretor(e). 
Avant de finir cette présentation sommaire des projets d'UZEI dans le domaine de la lexicographie assistée par ordinateur, nous voudrions revenir sur les difficultés de lemmatisation évoquées plus haut. La lemmatisation «manuelle» ou semi-automatique demande un grand effort que nous voudrions alléger et c'est dans ce but que nous voulons créer EUSLEM, un lemmatiseur automatique basé sur l'analyse morphologique du basque (basé, à son tour, sur la morphologie de deux niveaux, cf. Koskenniemi 1983). C'est un projet à développer en collaboration avec la Faculté d'Informatique de l'Université du Pays Basque comme suite à l'analyseur et au correcteur d'orthographe.

Nous présentons ici un schéma des cas auxquels doit faire face le programme:

\begin{tabular}{|c|l|c|c|c|l|}
\hline $\begin{array}{c}\text { Forme } \\
\text { normalisée }\end{array}$ & Forme erronée & $\begin{array}{c}\text { Forme étudiée } \\
\text { par le lexique } \\
\text { général } \\
\text { (lemme de la } \\
\text { variable) }\end{array}$ & $\begin{array}{c}\text { Fautes } \\
\text { typiques } \\
\text { (règles) }\end{array}$ & $\begin{array}{c}\text { Variable/fautes } \\
\text { typiques } \\
\text { étudiées par } \\
\text { le lexique }\end{array}$ & $\begin{array}{l}\text { Exemple (mot-texte, lemme } \\
\text { standard, lemme de la variable, } \\
\text { catégorie grammaticale) }\end{array}$ \\
\hline+ & + & & & (testuko testu testu IZE) \\
\hline+ & & - & & - & (afasiagatik ? afasia IZE) \\
\hline- & & - & & + & (baltzaren beltz baltz ADJ) \\
\hline- & & - & & - & (axtelehenean ? axtelehen IZE) \\
\hline- & & - & + & - & (zuaitzeko zuhaitz zuaitz IZE) \\
\hline+ & + & - & & - & (extean ? exte IZE)* \\
\hline
\end{tabular}

Dans sa configuration actuelle, ce lemmatiseur devrait être capable d'étudier les formes simples. Pour qu'il puisse traiter des formes complexes, une nouvelle démarche s'impose; elle doit commencer par l'élaboration d'une typologie de ces formes complexes, mais nous n'en sommes qu'aux rudiments.

\section{Note}

* Cet article est issu d'une communication présentée par l'auteur aux IVes Journées scientifiques du réseau «Lexicologie, terminologie, traduction» de l'AUPELF-UREF (Lyon, France, 28, 29, 30 septembre 1995). 


\section{RÉFÉRENCES}

ADURIZ, I., ALEGRIA, I., ARRIOLA, J. M., ARTOLA, X., DIAZ DE ILARRAZ, A., EZEIZA, N., GOJENOLA K. et M. MARITXALAR (1995) : «Different Issues in the Design of a Lemmatizer/Tagger for basque. "From Text to Tag"», SIGDAT, EACL

ADURIZ, J. A. et M. URKIA (1994) : «Hiztegi Batuaren Datu-Base Lexikografikoa», Euskera (Actes du Congrès d'Euskaltzaindia), Leioa, Euskaltzaindia.

AGIRRE, E., ALEGRIA, I., ARREGI, X., ARTOLA, X., DIAZ DE ILARRAZ, A., SARASOLA, K. et M. URKIA (1989): «Aplicación de la morfología de nos niveles al euskara», SEPLN, vol. 8, Barcelone, pp. 87-102.

ALDEZABAL, I., ALEGRIA, I., ARTOLA, X., DIAZ DE ILARRAZ, A., EZEIZA, N., GOJENOLA, K., ADURIZ I. et M. URKIA (1994) : «EUSLEM: Un lematizador/etiquetador de textos en euskara, SEPLN, Córdoba.

DA COSTA, A. (1989): RTerm2, HIZKIA, Bayonne.

Euskaltzaindia (1985, 1990) : Euskal Gramatika: Lehen urratsak (I, II eta III), Bilbo, Euskaltzaindia.

Euskaltzaindia (1992): Euskaltzaindiaren Gomendioak eta Erabakiak (I eta I), Bilbo, Euskaltzaindia.

KOSKENNIEMI, K. (1983) Two-Level Morphology: A general Computational Model for Word-Form Recognition and Production, University of Helsinki, Department of General Linguistics, Publication 11.

Oracle Corporation (1993): ORACLE SQL*Forms, SQL*Plus, PL / SQL, Text*Retrieval and so on, Redwood City.

SAGARNA, A. et M. URKIA (1991): «Terminología y Lexicografía asistida por ordenador. La experiencia de UZEI», SEPLN, vol. 8, Donostia. 